Running head: Multisensory integration in tennis

\title{
Auditory contributions to visual anticipation in tennis
}

\author{
Rouwen Cañal-Bruland ${ }^{1}$, Florian Müller ${ }^{1}$, Björn Lach $^{1}, \&$ Charles Spence ${ }^{2}$ \\ ${ }^{1}$ Department of Sport Psychology, Institute of Sport Science, Friedrich Schiller University \\ Jena, Germany \\ ${ }^{2}$ Crossmodal Research Laboratory, Department of Experimental Psychology, Oxford \\ University, United Kingdom
}

\section{Corresponding author:}

Rouwen Cañal-Bruland, Institute of Sport Science, Friedrich Schiller University Jena, Seidelstraße 20, 07749 Jena, Germany; Email: rouwen.canal.bruland@uni-jena.de 


\begin{abstract}

\section{Objectives}

It is well-established that visual information is crucial to successful anticipation in
\end{abstract} tennis. Here, we examine whether the sound of a tennis ball being struck by a racquet would influence anticipatory judgments of its trajectory.

Design

Within videos of tennis rallies from the semi-final of the Australian Open 2016 between Djokovic and Federer, the intensity of the sounds produced when the racquet hit the ball were systematically manipulated.

\title{
Methods
}

Experienced tennis players had to anticipate where the ball would land in the opponent's half.

Results

The results highlight that the louder the sound of the contact between the racquet and ball, the longer participants predicted the ball's trajectory to be.

\section{Conclusion}

These findings demonstrate that besides the crucial contribution of vision to anticipation, action-related auditory cues significantly influence predictions of the outcome of strokes in tennis as well.

Key words: Auditory and visual perception, multisensory integration, anticipation, action sounds, sport

Word count: 2079 words (includes everything apart from the abstract; no more than 13 pages) 
Auditory contributions to visual anticipation in tennis

After playing the inaugural match under the new roof of the Wimbledon centre court in 2009 Andre Agassi had the following to say: "This was amazing. The way the ball sounds in here is going to add so much intensity for the players” (BBC News, May 17, 2009). Such a statement suggests that auditory information may influence a tennis player's performance. Thus far, however, researchers have focused primarily on the pick-up of visual information when examining, for example, the perceptual information contributing to anticipatory judgments in racquet sports such as tennis (for recent reviews, see Cañal-Bruland \& Mann, 2015; Loffing \& Cañal-Bruland, 2017; but see Sinnett \& Kingstone, 2010, for an exception). Here, we report on a study demonstrating that auditory information influences people's predictions of the outcome of an opponent's tennis strokes. That is, we provide evidence that the louder the sound that results from the contact between racquet and ball, the longer participants predict the ball's trajectory to be. Such results are consistent with current theories highlighting the multisensory nature of human perception (see Bremner, Lewkowicz $\&$ Spence, 2012; Stein, 2012, for reviews). Moreover, this prediction is in line with a recent study that examined the roles of auditory and visual information in discriminating the power of, among other things, volleyball smashes. In fact, Sors et al. (2017) showed that auditory information led to more accurate discrimination judgements about the balls' speed than visual information, thereby providing initial evidence for the sensitivity of the auditory system when it comes to judging the power of impact and consequently the ball's resulting speed.

In tennis, to date only two studies have been published that have examined whether auditory information adds to the pick-up of crucial visual information when anticipating the outcome of an opponent's strokes (Mead \& Drowatzky, 1997; Takeuchi, 1993). Both studies reported that withholding auditory information had a detrimental effect on a player's performance. Yet, as both studies followed an all-or-nothing principle, that is, they either 
completely withheld auditory information or else provided normal auditory and visual information, it remains unclear whether the loudness of the racquet-ball contact may systematically influence viewers' predictions of tennis strokes.

The aim of the current study was to examine whether the sound of a tennis ball being struck by a racquet would influence anticipatory judgments of its trajectory. To this end, we presented experienced tennis players with videos of tennis rallies in which the intensity of the sounds produced when the racquet hit the ball were systematically manipulated. The participants had to judge where the ball would land in the opponent's half. We hypothesized that the louder the sound of the contact between the racquet and ball, the longer participants would predict the ball's trajectory to be.

\section{Method}

We tested $23^{1}$ experienced tennis players ( $M=30.0$ years; $S D=11.4,5$ females $)$. Participants had a mean playing experience of 18.7 years $(S D=10.9)$ with a minimum of at least 5 years of playing experience. The participants were individually presented with 165 videos $(720 \mathrm{p}, 25 \mathrm{fps}$, mean length $=1980 \mathrm{~ms} ; \mathrm{SD}=400 \mathrm{~ms})$ showing snippets from tennis rallies from the semi-final of the Australian Open 2016 between Djokovic and Federer (available on youtube.com; for a representative snapshot from the footage, see Figure 1, Top), on a laptop (ASUS X553M) with a 15.6 inch display (1366 x 768 pixels). They had to predict where the ball would land in the opponent's half of the court (always displayed in the lower half of the screen, see Figure 1, Top). To mark their predictions, immediately after the video had finished participants were presented with a sketch of the lower half of the court that was identical in size to the one they had just seen in the video (see Figure 1, Bottom). In this sketch, the participants used a mouse to move a cursor on the screen to indicate their predicted landing location, and then saved their answer by clicking the mouse button. The 
videos were occluded immediately after any sound resulting from the racquet-ball contact had ceased. The actual landing positions were distributed as illustrated in Figure 1 (Top), and the videos were presented in an individually randomized order. The experiment was implemented using standard web technologies (html, javascript) and presented via the Opera browser (www.opera.com).

Using audio/video editing software (Audacity, XMedia Recode), we manipulated the sound intensity of the racquet-ball contact of the to-be-judged strokes in one of two directions, either increasing or decreasing the intensity of the sound by $12 \mathrm{~dB}$ within each of 55 original video clips. Together with the original videos this resulted in a total of $55 \times 3=$ 165 videos, representing the three levels of the within participant factor sound intensity (low, normal, high). Note that we did not determine the actual sound intensity for each original clip, but that the systematic manipulation of the audio tracks for each clip does not hinge on knowing the actual loudness.

This main experiment was followed by a manipulation check, requiring participants to rate $20(10 x-12 \mathrm{~dB}$ and $10 \mathrm{x}+12 \mathrm{~dB})$ randomly presented video clips on a 7 point Likertscale for the intensity of the displayed stroke (i.e., how hard the ball had been struck; ranging from not at all to extremely hard).

The study concluded with an exit questionnaire collecting information about demographics (including age, gender etc.), playing experience (e.g., years of playing experience), as well as questions concerning whether participants identified the purpose and manipulations of the study. This questionnaire revealed that none of the participants was aware of the sound manipulation during the main experiment.

*** Insert Figure 1 about here *** 


\section{Results}

To test whether changes in sound intensity systematically influenced participants' anticipatory judgments in the longitudinal axis, we subjected the predicted longitudinal ball coordinates to an ANOVA with the within-subject factor Sound Intensity (-12db, original, $+12 \mathrm{db})$. The results confirmed a significant effect of sound intensity, $F(2,44)=29.39, p<$ .001 (Greenhouse-Geisser corrected), $\eta_{p}{ }^{2}=.57$. Figure 2 illustrates that the louder the ballracquet contact sound, the longer participants predicted the ball's trajectory to be. Bonferroni corrected post-hoc comparisons indicated that strokes with less intense sounds $(-12 \mathrm{~dB})$ resulted in significantly shorter trajectories than normal (unmanipulated) sounds, $t(22)=4.66$, $p<.001, d=0.97$, whereas strokes with more intense (i.e., louder) sounds $(+12 \mathrm{~dB})$ resulted in significantly longer trajectories than the unmanipulated strokes, $t(22)=4.02, p<.002, d=$ 0.84. Consequently, more intense sounds yielded significantly longer trajectories than less intense sounds, $t(22)=6.34, p<.001, d=1.32$ (for the mean predicted ball landing positions for the low and high sound intensity conditions, see also Fig. 1, Bottom). Neither the radial errors (overall accuracy, $p=.30$ ) nor the predicted horizontal position $(p=.95)$ were affected by sound intensity. In addition, results concerning the manipulation check confirmed that participants judged more intense ball-racquet contact sounds to have been struck harder than less intense ball-racquet contact sounds, $t(22)=8.39, p<.001, d=1.75$.

$* * *$ Insert Figure 2 about here $* * *$

\section{Discussion}

Taken together, these results demonstrate that auditory information can significantly influence anticipatory judgments of the outcome of a player's strokes in tennis in a predictable manner. The more intense the racquet-ball contact sound, the longer participants 
predicted the ball's trajectory to be. Notably, this effect occurred implicitly, as participants were unaware of the fact that racquet-ball contact sounds had been manipulated.

From a practical viewpoint, these findings have several implications: First, while there is strong evidence that the pick-up of visual information is crucial to anticipatory judgments in racquet sports such as tennis (e.g., Loffing \& Cañal-Bruland, 2017), this study provides evidence to suggest that sound intensity is clearly taken into account when trying to predict the consequences of an opponent's strokes (for similar findings in e.g. volleyball, see Sors et al., 2017). It follows that going beyond earlier studies that focused on the visual cues needed to successfully predict tennis strokes (e.g., Ward, Williams \& Bennett, 2002), we provide evidence that a multisensory approach promises to be more successful.

Second, because sound intensity systematically and implicitly biases an opponent's judgments about the outcome of a stroke ("the more intense, the longer the trajectory"), players may think of asking their racquet manufacturers to produce racquets that deliver the same performance, but to deliberately manipulate sound intensities to their own ('invisible') advantage. A similar, but certainly more explicit technique that has been shown to negatively influence an opponent's performance is to divert his/her attention by grunting (Sinnett \& Kingstone, 2010).

Finally, the results also speak to current theories of multisensory integration (see Bremner et al., 2012; Stein, 2012). In particular, they add to a growing body of research showing that our perception of everything from the crunch of potato chips and apples through to the carbonation of a beverage held in the hand is influenced by associated auditory information (Demattè et al., 2014; Zampini \& Spence, 2004, 2005; see Spence \& Zampini, 2006, for a review). 


\section{References}

Bremner, A., Lewkowicz, D., \& Spence, C. (Eds.). (2012). Multisensory development. Oxford, UK: Oxford University Press.

Cañal-Bruland, R., \& Mann, D. L. (2015). Time to broaden the scope of research on anticipatory behavior: A case for the role of probabilistic information. Frontiers in Psychology, 6:1518. (doi:10.3389/fpsyg.2015.01518)

Demattè, M. L., Pojer, N., Endrizzi, I., Corollaro, M. L., Betta, E. Aprea, E., Charles, M., Biasioli, F., Zampini, M., \& Gasperi, F. (2014). Effects of the sound of the bite on apple perceived crispness and hardness. Food Quality and Preference, 38, 58-64. (doi:10.1016/j.foodqual.2014.05.009)

Loffing, F., \& Cañal-Bruland, R. (2017). Anticipation in sport. Current Opinion in Psychology, 16, 6-11. (doi:10.1016/j.copsyc.2017.03.008)

Mead, T. P., \& Drowatzky, J. N. (1997). Interdependence of vision and audition among inexperienced and experienced tennis players. Perceptual and Motor Skills, 85, $163-$ 166. (doi:10.2466/pms.1997.85.1.163)

Sinnett, S., \& Kingstone, A. (2010). A preliminary investigation regarding the effect of tennis grunting: Does white noise during a tennis shot have a negative impact on shot perception? PLoS ONE 5(10):e13148. (doi:10.1371/journal.pone.0013148)

Sors, F., Murgia, M., Santoro, I., Prpic, V., Galmonte, A., \& Agostini, T. (2017). The contribution of early auditory and visual information to the discrimination of shot power in ball sports. Psychology of Sport and Exercise, 31, 44-51.

(doi:10.1016/j.psychsport.2017.04.005) 
Spence, C., \& Zampini, M. (2006). Auditory contributions to multisensory product perception. Acta Acustica united with Acustica, 92, 1009-1025.

Stein, B. E. (Ed.). (2012). The new handbook of multisensory processing. Cambridge, MA: MIT Press.

Takeuchi, T. (1993). Auditory information in playing tennis. Perceptual and Motor Skills, 76, 1323-1328. (doi:10.2466/pms.1993.76.3c.1323)

Ward, P., Williams, A. M., \& Bennett, S. J. (2002). Visual search and biological motion perception in tennis. Research Quarterly for Exercise and Sport, 73, 107-112. (doi:10.1080/02701367.2002.10608997)

Zampini, M., \& Spence, C. (2004). The role of auditory cues in modulating the perceived crispness and staleness of potato chips. Journal of Sensory Science, 19, 347-363. (doi:10.1111/j.1745-459x.2004.080403.x)

Zampini, M., \& Spence, C. (2005). Modifying the multisensory perception of a carbonated beverage using auditory cues. Food Quality and Preference, 16, 632-641. (doi:10.1016/j.foodqual.2004.11.004) 


\section{Footnotes}

${ }^{1}$ In the absence of previous research on this subject, sample size was determined on the basis of a medium sized effect $(d=.6)$ and targeting a power of .8 for a one-tailed withinparticipant comparison of means between the low and high sound intensity conditions. This necessitated a sample size of at least $N=19$ participants. Note, further, that in total 24 participants were recruited with one participant failing to finish the experiment due to a problem with data collection. All of this participant's data were removed from any further analyses, resulting in a final sample size of $N=23$. 
Figure Captions

Figure 1. Top) representative snapshot taken from the experimental footage including the distribution of the actual ball landing positions. Bottom) Sketch of the lower half of the court that was identical in size to the one seen in the video and used to mark the participants' responses. White dots represent the mean predicted landing positions for each clip in the low intensity condition; black dots represent the mean predicted landing positions for the corresponding clip in the high intensity condition.

Figure 2. The influence of sound intensity on anticipatory judgments of the ball's landing position in the longitudinal (i.e., vertical) axis (higher pixel values indicate longer predicted trajectories in the direction of the baseline). Error bars indicate $95 \%$ confidence intervals. 
Figures

Figure 1.

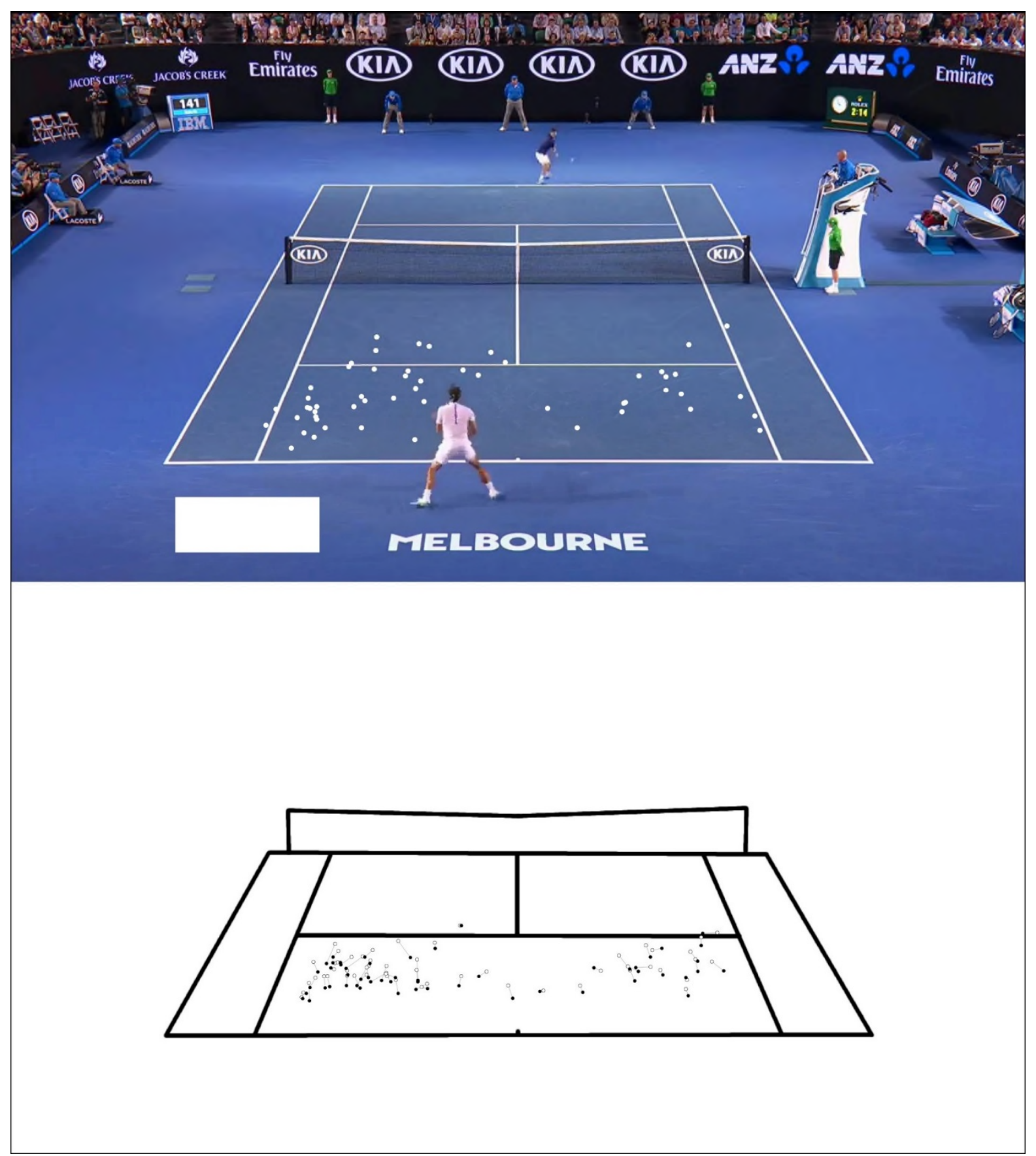


Figure 2.

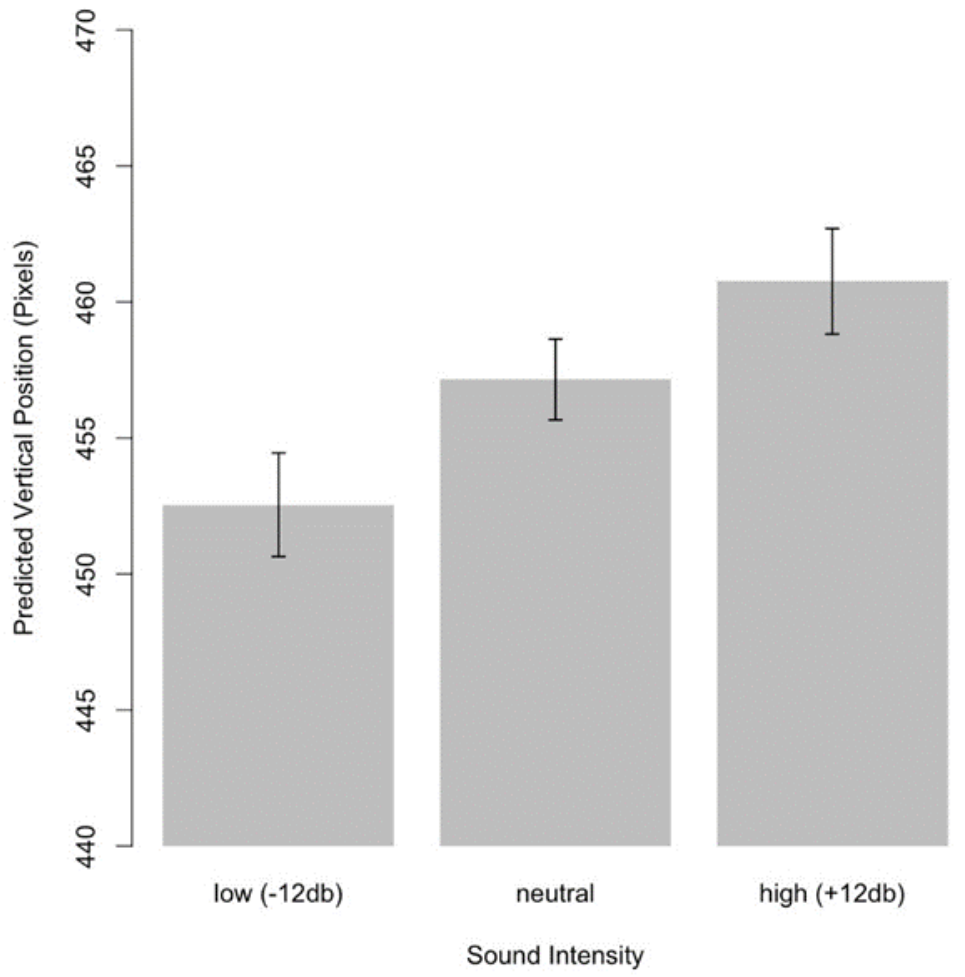

